Supporting Information

\title{
Allele-Specific RNA Knockdown with a Biologically Stable and Catalytically Efficient XNAzyme
}

Kim Nguyen ${ }^{1 \dagger}$, Yajun Wang ${ }^{1 \dagger}$, Whitney E. England ${ }^{1}$, John C. Chaput ${ }^{1,2,3^{*}}$, and Robert C. Spitale $1,2,3 *$

(1) Department of Pharmaceutical Sciences, (2) Department of Molecular Biology \& Biochemistry and (3) Department of Chemistry. University of California, Irvine. Irvine, California. 92697 USA

† Signifies co-first author status

* To whom correspondence should be addressed:

Robert C. Spitale

Department of Pharmaceutical Sciences

University of California, Irvine

Irvine, CA 92697-3958

(t) 949-824-7655

(e) rspitale@uci.edu

John C. Chaput

Department of Pharmaceutical Sciences

University of California, Irvine Irvine, CA 92697-3958

(t) 949-824-8149

(e) jchaput@uci.edu

ORCIDs JC: 0000-0003-1393-135X

RS: $\quad 0000-0002-3511-8098$ 


\section{Table of Contents}

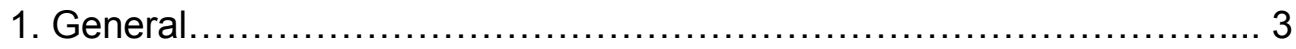

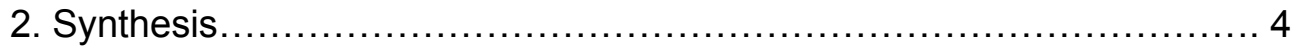

3. Biochemical Methods............................................. 4 - 8

4. Supplementary Figures............................................. 9 - 13

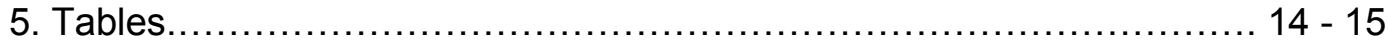

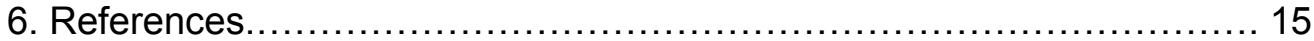




\section{General}

2'F-araNTPs (faATP, faCTP, faGTP, faUTP) were obtained from Metkinen Chemistry (Kuusisto, Finland). DNA, FANA, and 5'-hexynyl phosphoramidites, as well as Universal Support II CPG columns were purchased from Glen Research (Sterling, Virginia). TNA phosphoramidites were synthesized in-house following procedures reported previously. Oligonucleotides containing FANA and TNA were synthesized on an ABI3400 DNA synthesizer using chemical synthesis reagents purchased from Glen Research (Sterling, Virginia). DNA and RNA oligonucleotides were purchased from Integrated DNA Technologies (Coralville, IA). All oligonucleotides were purified by denaturing polyacrylamide gel electrophoresis, and quantified by UV absorbance. YM-3 microcentrifugal concentrators were purchased from EMD Millipore (Billerica, MA). 


\section{Synthesis of XNA oligonucleotides}

XNA containing oligonucleotide synthesis and preparation.

TNA phosphoramidites were synthesized as reported previously. Standard $\beta$-cyanoethyl phosphoramidite chemistry and an Applied Biosystems 3400 DNA Synthesizer were used to synthesize TNA oligonucleotides on Universal Support II CPG columns in $1 \mu$ mole scale. Standard DNA coupling procedures were modified for FANA and/or TNA containing oligonucleotides such that coupling time for FANA and TNA amidites was increased to $360 \mathrm{~s}$ and $1200 \mathrm{~s}$, respectively. Detritylation was performed in two cycles for TNA amidites, $60 \mathrm{~s}$ each. Oligonucleotides used for biostability studies were coupled with a 5'-hexynyl phosphoramidite for later IR-680 fluorophore tagging via click chemistry. Cleavage from the solid support and final deprotection of oligonucleotides synthesized were achieved simultaneously in $\mathrm{NH}_{4} \mathrm{OH}(33 \%)$ for $18 \mathrm{~h}$ at $55^{\circ} \mathrm{C}$. Oligonucleotides were purified on denaturing (8 M urea) PAGE, recovered by electro-elution, and subsequently desalted by buffer exchange using microcentrifugal concentrators, and quantified by nano-drop. All oligonucleotides synthesized in-house were subjected to Quadrupole time-of-flight mass spectrometry (Q-TOF) for identity confirmation.

\section{Biochemical methods and data analysis}

In vitro RNase $H$ activity assays with $X 10-23$, inactive $X 10-23$, and $X$-antisense oligos targeting RNA substrates of KRAS WT or KRAS G12C.

All RNase $\mathrm{H}$ activity assays were performed under simulated physiological buffer conditions in 50 $\mathrm{mM}$ Tris- $\mathrm{HCl}\left(\mathrm{pH} \mathrm{7.5)} \mathrm{containing} 0.5 \mathrm{mM} \mathrm{MgCl}_{2}, 150 \mathrm{mM} \mathrm{NaCl}\right.$, and 0.1 unit/ $\mu \mathrm{L}$ of RNase $\mathrm{H}$ at $37^{\circ} \mathrm{C}$. $0.5 \mu \mathrm{M}$ of each of the KRAS WT and KRAS G12C RNA substrate were mixed with $1 \mu \mathrm{M}$ of the tested constructs of $\mathrm{X} 10-23$, inactive $\mathrm{X} 10-23$, and $\mathrm{X}$-antisense oligo in Tris- $\mathrm{HCl}(\mathrm{pH} 7.5)$ buffer, respectively, to anneal by heating for $5 \mathrm{~min}$ at $90^{\circ} \mathrm{C}$ and cooling for $5 \mathrm{~min}$ on ice. Reactions were initiated by the addition of $\mathrm{MgCl}_{2}, \mathrm{NaCl}$, and $\mathrm{RNase} \mathrm{H}$ to the final concentration. Reactions were sampled by quenching $1.51 .5 \mu \mathrm{L}$ of reactions using $15 \mu \mathrm{L}$ (10 equivalents, v/v) of formamide containing $25 \mathrm{mM}$ EDTA at time points of $0,1,5$, and 20 hours. Samples were denatured for $15 \mathrm{~min}$ at $95^{\circ} \mathrm{C}$ and analyzed by $15 \%$ denaturing PAGE. Gels were visualized using a LI-COR Odyssey CLX.

\section{Cloning and plasmids.}

The wild type (WT) KRAS gene (transcript variant d), which encodes a protein annotated as GTPase Kirsten rat sarcoma viral proto-oncogene isoform b from Homo sapiens (U.S. NCBI Accession \#: NP_001356716.1; UniProtKB: Q01116) was reverse transcribed from total RNA of HeLa cells and amplified by PCR to generate a Ndel-BamHl fragment for cloning downstream of GFP coding sequence (pCDNA3.3-EGFP) resulting a fusion of EGFP-WT-KRas (pKN-CMVEGFP-WT-KRAS). G12V-KRAS mutant was generated by site-directed mutagenesis PCR using pKN-CMV-EGFP-WT-KRAS as the template and subsequently amplified as Ndel-BamHI fragment for cloning downstream of mCherry coding sequence in pKN-CMV-mCherry resulting fusion of mCherry-G12V-KRAS (pKN-CMV-mCherry-G12V-KRAS). Sequence quality of both fusion constructs were verified by sequencing analysis via Genewiz Inc. sequencing services

\section{Cell lines, bacterial and mammalian culture conditions.}


HEK293T (HEK) and HeLa cells were cultured in DMEM (Corning, Cat\#: 10-017-CM) supplemented with $10 \% \mathrm{FBS}, 1 \%(1 \mathrm{mg} / \mathrm{mL})$ penicillin and streptomycin and grown at $37 \circ \mathrm{C}, 5 \%$ $\mathrm{CO}_{2}$. NCl-H441 (G12V KRAS) cells (ATTCC, Cat\#: ATCC-CRM-HTB-174) were cultured in ATCC-formulated RPMI-1640 (ATCC, Cat\#: ATCC3-2001) supplemented with 10\% FBS, 1\% (1 $\mathrm{mg} / \mathrm{mL}$ ) penicillin and streptomycin.

pCDNA3.3-EGFP, pKN-CMV-EGFP-WT-KRAS and pKN-CMV-mCherry-G12V-KRAS were used to transform $E$. coli NEBStable cells and grown in LB medium at $37^{\circ} \mathrm{C}$ with ampicillin at $100 \mu \mathrm{g} / \mathrm{mL}$.

\section{Transfection.}

For single KRAS $\times 10-23$ experiments in NCl-H441 cells to assess targeting specificity: After $96 \mathrm{~h}$ seeding of $2.5 \times 10^{5}$ cells/well of $\mathrm{NCl}-\mathrm{H} 441$ cells in 6 -well plates, the cells were transfected with transfection carrier only (Negative control) or with $500 \mathrm{nM}$ of G12V KRAS X10-23 for 96h using JetPrime Transfection reagent (Polyplus Transfection, France) according to manual instruction except adding $90 \mu \mathrm{L}$ of JetPrime Reagent per well. For negative controls, the volume of JetPrime Reagent used for each well was the same as those with X10-23 to ensure the same transfection condition in the control and experimental samples. Parameters used in RNA isolation at $96 \mathrm{~h}$ post-transfection are the same as described in RNA isolation section.

For benchmark experiments: After $96 \mathrm{~h}$ seeding at $2.5 \times 10^{5}$ cells/well of NCl-H441 cells in 6 -well plates, the cells were transfected with transfection carrier only (Negative control-0) or $16 \mu \mathrm{g}(306.81 \mathrm{nM})$ of G12V Kras X10-23, 16 $\mu \mathrm{g}(604.28 \mathrm{nM})$ of the corresponding antisense (ASO), or 16 $\mathrm{gg}(305.71 \mathrm{nM})$ of internal GFP X10-23 which was also served as negative controls using JetPrime Transfection reagent (Polyplus Transfection, France) according to manual instruction except adding $5 x$ higher than the manual recommended volume of JetPrime Reagent. After $96 \mathrm{~h}$ post transfection, cells were subjected to RNA isolation as described in RNA isolation section.

\section{RNA isolation and reverse transcription (RT).}

To each well of the 6-well plate of cells (HEK293T or NCl-H441), total RNA isolation was performed using $1 \mathrm{~mL} /$ well Trizol Reagent (Invitrogen) according to the manufacturer instructions. Total RNA was treated with Turbo DNAse (20U/reaction) at $37^{\circ} \mathrm{C}$ for $30 \mathrm{~min}$ on shaker, and followed by purification using equal volume of Phenol-Chloroform, $\mathrm{pH} 4.5$ (Thermal Fisher, Ambion Cat\#:AM9720). Aqueous layer was transferred to a new tube and precipitated with one tenth volumes of $5 \mathrm{M} \mathrm{NaCl}$ and one volume of isopropanol at $-20^{\circ} \mathrm{C}$ overnight. Precipitated RNA was pelleted at $4{ }^{\circ} \mathrm{C}$ and $15000 \mathrm{rpm}$ using bench-top centrifuge and followed by two washes with cold $\left(-20^{\circ} \mathrm{C}\right) 70 \%$ ethanol. Two microgram $(2 \mu \mathrm{g})$ of DNA-free RNA was subjected to cDNA synthesis using SuperScript III First-strand Synthesis System (Invitrogen-Life Technologies, CA) with random hexamer primers in a $20 \mu \mathrm{L}$ reaction according to the manufacturer instructions. cDNA was subsequently purified using DNA Clean \& Concentration columns from Zymo Research (Cat\# D4003) according to the manufacturer instructions and eluted 2x with $100 \mu \mathrm{L}$ water/each.

\section{SYBR Green semi-quantitative PCR (qPCR) analysis.}

To quantify copy number of GFP transcript in the presence or absence of GFP-X10-23, cDNA was subjected to $\mathrm{qPCR}$ analysis using $\mathrm{iQ}(\mathrm{tm}) \operatorname{SYBR}(\mathrm{R})$ Green Supermix (BioRad, Cat\# 1708880) on BioRad CFX real time PCR system. In each qPCR run, known concentration of DNA standards 
at 5 serial dilutions was used to establish standard curve and calculation of starting quantity (SQ) of target transcripts. Specific primers for interrogating EGFP, mCherry, KRAS, and twenty five offtargets, GAPDH (loading control) transcripts as well as for qPCR Standards are listed in Table S1. For each experimental sample, three replicates were performed, and each serial diluted Standard was assayed in duplicates. Relative mRNA copy number of target transcripts was calculated by multiplying individual $S Q$ to a corresponding scaling factor derived from loading control GAPDH SQ. By dividing the median of GAPDH SQ in a qPCR run to individual GAPDH $S Q$, a scaling factor for that particular sample was generated.

\section{Targeting specificity analysis of X10-23 via single/co-culture of WT \& G12V KRAS.}

Single culture: HEK293T $\left(5 \times 10^{5}\right)$ cells seeded in 6-well plate. Thirty-six hour post seeding, HEK293T cells were transfected with (a) $1 \mu \mathrm{g}$ pKN-CMV-EGFP-WT-KRAS + $8 \mu \mathrm{g}$ WT-KRAS X1023, (b) $1 \mu \mathrm{g}$ pKN-CMV-EGFP-WT-KRAS + $8 \mu \mathrm{g}$ G12V-KRAS X10-23, (c) $1 \mu \mathrm{g}$ pKN-CMVmCherry-G12V-KRAS + $8 \mu \mathrm{g}$ WT-KRAS X10-23, or (d) $1 \mu \mathrm{g}$ pKN-CMV-mCherry-G12V-KRAS + $8 \mu \mathrm{g} \mathrm{G12V-KRAS} \mathrm{X10-23} \mathrm{(} 8 \mu \mathrm{g}$ of WT or G12V KRAS X10-23 is equivalent to $378.57 \mathrm{nM}$ ) using JetPrimer Transfection reagent (Polyplus Transfection, France). At 144h post transfection, the cells were subjected to RNA extraction using Trizol reagents, followed by DNAse treatment and RT-qPCR analysis using unique primers for each target. For EGFP-WT-KRAS transcript, EGFP primers: oKN424F and oKN421R were used. For mCherry-G12V-KRAS target, mCherry primers: oKN458F and oKN459R were used. For loading control, GADPH primers: oKN60F and oKN61R were used.

Co-cultures: Cells were transfected with the same experimental settings used in single culture. However, at 48h post transfection, cells were trypsinized to detach from plate and pooled as such equal number of cells in (a) and (c) were combined; equal number of cells in (b) and (d) were combined [See the Single culture section above for description of (a) to (d) conditions]. After 96h re-seeding (144h post transfection), the co-cultures were subjected to RNA isolation, DNAse treatment, RT-qPCR analysis using unique primers for each target and loading control as described above.

\section{RT-PCR-Restriction Fragment Length Polymorphism Assay (PCR-RFLPA)}

In NCl-H441 cells (papillary adenocarcinoma) there are 4 genomic copies of G12V to WT KRAS. To distinguish between the two allele mRNAs we optimized a PCR restriction fragment length polymorphism assay (PCR-RFLP) ${ }^{1}$ which was previously described in Sunaga et al., (2011) Mol. Canc. Therapy. PCR-RFLP was used to visually differentiate between WT and G12V KRAS alleles. First, we introduce a mutation ( $G$ to $C$ ) via a primer used in the amplification of KRAS target at the proximity of G12V mutation, such that only WT allele acquires a recognition sequence of $B s t N I$ restriction enzyme. PCR product was digested with BstNI and subjected to gel electrophoresis and visualized by staining. Amplicons derived from WT allele was then cleaved resulting a smaller DNA fragment (bottom band) than those derived from G12V allele when resolved on agarose gel. Here how the assay was performed: In brief, cDNA is amplified by PCR using a unique sense primer that carries a single substitution ( $G$ to $C$ ) at the first residue of KRAS codon 11 (GCT to CCT). This substitution introduces BstNI (NEB, USA; Cat\# R0168S) recognition site ("CCWGG"), which spans from KRAS codon 11 (CCT) to codon 12 (GGT), in WT KRAS but not the G12V allele harboring one mutation at codon 12 (GGT to GTT). Thus, upon BstNI digest, amplicons from WT allele will be cleaved and leave G12V mutant allele untouched, resulting digested amplicons of WT allele having 36nt shorter than G12V mutant. In this study, the sense primer from Sunaga et al. is modified by adding extra 14nt (lower case) at the 5'end, resulting an increase in size difference of the two alleles by 50nt upon BstNI digest (oKN484F: 5'ttattataaataatGACTGAATATAAACTTGTGGTAGTTGGAcCT-'3). Lower case "c" in oKN484F

denotes a mutation introduced in the primer to create BstNI recognition sequence. Two micrograms $(2 \mu \mathrm{g})$ of DNA-free RNA from controls, (-) X10-23, as well as experimental, (+) X10- 
23, samples were subjected to cDNA synthesis using SuperScript III First-strand Synthesis System (Invitrogen-Life Technologies, CA) with random hexamer primers in a $20 \mu \mathrm{L}$ reaction according to the manufacturer instructions. Ten microliters of purified cDNA (1:100dilution) were used as DNA template for PCR using DreamTaq DNA polymerase (Fisher Scientific, Cat\# FEREP0702) with oKN484F and the original antisense primer (oKN483R: 5'TCCTCTTGACCTGCTGTGTCG-'3) in 50uL reaction. PCR thermal parameters used: $1 \times 94^{\circ} \mathrm{C}$ $2 \mathrm{~min}, 40 \mathrm{x}\left(94^{\circ} \mathrm{C}-20 \mathrm{sec}, 58^{\circ} \mathrm{C}-15 \mathrm{sec}, 70^{\circ} \mathrm{C}-15 \mathrm{sec}\right), 1 \times 70^{\circ} \mathrm{C}-5 \mathrm{~min}$. Four controls used for comparison migration patterns of digested amplicons of WT vs. G12V alleles. For WT allele: there are pKN-CMV-EGFP-WT-KRAS plasmid and endogenous KRAS transcript from HeLa (WT allele). For G12V allele: there are pKN-CMV-mCherry-G12V-KRAS plasmid and endogenous KRAS transcripts from NCl-H441 cells which have heterozygous KRAS alleles (WT and G12V mutant). Endogenous KRAS transcript controls were processed the same way as mentioned above. For plasmid controls, 5ng DNA per reaction served as templates in PCR-RFLPA using oKN484F and oKN483R primers. Five hundred nanograms (500 ng) of PCR amplicons (controls and experimental) were digested with $30 \mathrm{U}$ of $B s t N I$ in $50 \mu \mathrm{L}$ digest reaction at $60^{\circ} \mathrm{C}$ for $3 \mathrm{~h}$. Digested applicons (132ng/well) were visualized on $3 \%$ agarose gels stained with SYBR Safe.

\section{Protein isolation and Immunoblot analysis.}

After $96 \mathrm{~h}$ seeding of $2.5 \times 10^{5}$ cells/well of $\mathrm{NCl}-\mathrm{H} 441$ cells in 6 -well plates, $\mathrm{NCl}-\mathrm{H} 441$ cells were transfected in duplicates with transfection carrier only or with either 51.6nM or 103nM G12VKRAS X10-23 per well. At 96h post transfection, cells were scraped off plates, divided in half (one for RNA isolation and the other for protein extraction) and spun down at $1000 \mathrm{xg}$ for $5 \mathrm{~min}$ at room temperature. Cell pellets subjected to protein isolation were re-suspended with $100-150 \mu \mathrm{L}(10 \mathrm{x}$ volume of dried cell pellet) RIPA lysis buffer [50mM Tris- $\mathrm{HCl}$ at $\mathrm{pH} 7.5,150 \mathrm{mM} \mathrm{NaCl}, 0.1 \%$ Triton, $0.5 \%$ Na-deoxycholate \& $0.1 \%$ SDS, $5 \mathrm{mM}$ EDTA), supplemented with $4 \mu \mathrm{L}$ of proteinase inhibitor cocktail Set VII (EMB, Cat\#: 539138-1ML), $4 \mu \mathrm{L}$ Turbo DNase (Life Tech., Cat\# AM2239), $4 \mu \mathrm{L}$ RNaseA (Thermo Fisher, Cat\# FEREN0531) and $40 \mu \mathrm{L}$ Halt Phosphorylase inhibitor (Fisher Scientific, Cat\# 78420) per milliliter of RIPA lysis buffer], was added. Cells were re-suspended and incubated on ice for $0.5 \mathrm{~h}$ then sonicated for 11 seconds ( 5 seconds ON, 1 second OFF) at $4^{\circ} \mathrm{C}$. Total protein lysate was cleared by centrifugation at $15 \mathrm{~K} \mathrm{rpm}$ for $30 \mathrm{~min}$ at $4^{\circ} \mathrm{C}$. The cleared lystate was subjected to BCA assay (BioSciences, Cat\# 786-570) to determine protein concentration according to manufacturer instruction at $37^{\circ} \mathrm{C}$ for $30 \mathrm{~min}$ and quantified using NanoDrop BCA program. SDS-PAGE analysis was performed: $30 \mu \mathrm{g}$ of total protein per well was resolved in 4-20\% gradient 10-well MiniProtean gel (Bio-Rad, Cat\# 4561094).

Proteins were transferred to nitrocellulose membrane using Trans-Blot Turbo Transfer unit (BioRad, Cat\#1704150). Membranes were blocked with 5\% BSA-Fraction V (Gold Biotechnology Inc, Cat\# A-420-25) in 1xTBST (1xTBS: $150 \mathrm{mM} \mathrm{NaCl}, 50 \mathrm{mM}$ Tris-HCl, pH 7.6 + 0.1\%Tween-20) for $1 \mathrm{~h}$ at room temperature and probed overnight at $4^{\circ} \mathrm{C}$ with fresh blocking buffer + rabbit anti-KRas (Proteintech, Cat\#: 12063-1-AP) at 1:2000dil or rabbit anti-MEK1/2 (Cell Signaling, MA, USA; Cat\# 8727S) at 1:1000dil or rabbit anti-phosphoMEK1/2 (Cell Signaling, MA, USA; Cat\# 2338S) at 1:2000dil or rabbit anti-beta-Actin (Cell Signaling, MA, USA; Cat\# 4967S) at 1:1000dil. Blot was washed $3 \times 10 \mathrm{~min} /$ each with $1 \times$ TBST at room temperature and incubated in 1xTBST+ 5\% BSAFraction $\mathrm{V}+$ anti-rabbit conjugated Horseradish peroxidase (1:20000 dilution) (Cell Signaling, MA, USA; Cat\# 7076S) for $1 \mathrm{~h}$ at RT. Finally, blot was washed $3 \times 10 \mathrm{~min} /$ each with $1 \times$ TBST at room temperature and imaged using ECL Chemiluminescent Substrate (Fisher Scientific, Cat\# PI32106) and imaged on a ChemiDoc MP imaging system (Bio-Rad).

\section{Analysis of the Cancer Genome Atlas Database and mutations.}

Mutation position, consequence, and affected case information for all single nucleotide polymorphisms was downloaded from The Cancer Genome Atlas Genomic Data Commons Data 
Portal release v19.0. For each gene impacted by a mutation, the sequences of two introduced dinucleotides resulting from the mutation (mutation plus one base up- or downstream) were established, and count of introduced dinucleotides was quantified across the entire mutation dataset. Cancer cases exhibiting mutations which introduced a GU dinucleotide were quantified across cancer types represented by TCGA projects. 


\section{Supplementary figures}

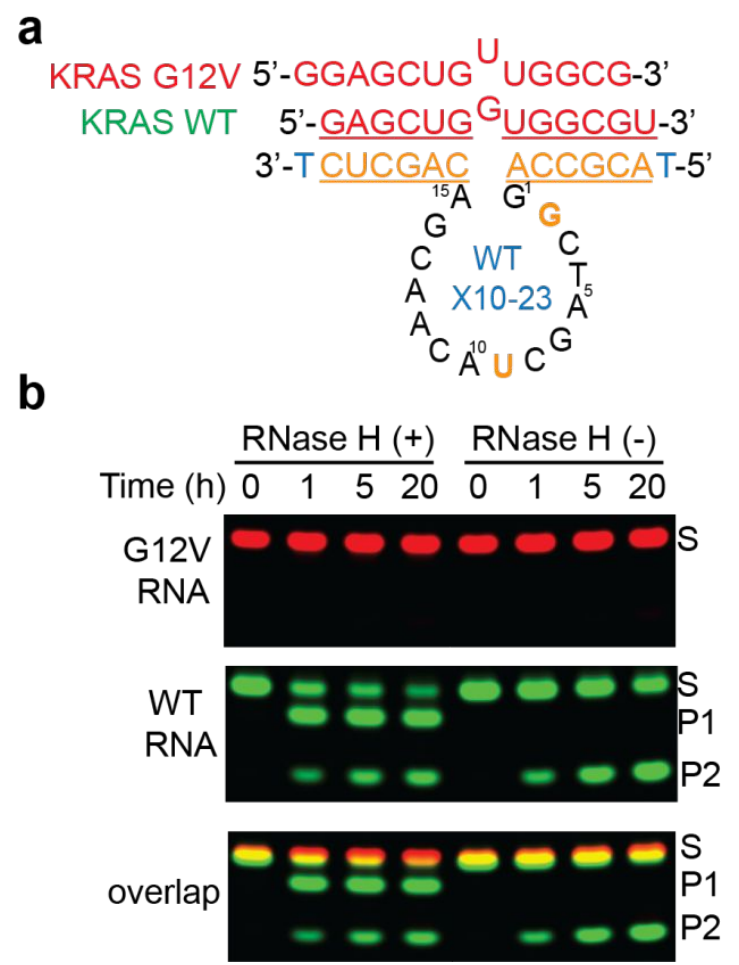

Figure S1. Allele-specific targeting of KRAS WT RNA substrate by catalytic X10-23 in vitro. a, X10-23 targeting KRAS WT RNA in complex with its RNA substrate with a cleavable unpaired $G$ positioned at the cleavage site, whereas when this X10-23 interacts with KRAS G12V substrate, an uncleavable $U$ is positioned at the cleavage site. $\mathbf{b}$, Representative PAGE images showing the RNA cleavage activity of X10-23 targeting the WT allele in the presence and absence of RNase $\mathrm{H}$. S: full-length substrate, P1: cleavage product from the activity of RNase H, P2: 5' cleavage product from the catalytic activity of X10-23. mRNAs of KRAS G12V and KRAS WT are shown in red and green, respectively. All assays were performed in buffers containing equimolar $(1 \mu \mathrm{M})$ of total RNA substrate (S) and X10-23 molecule, $0.5 \mathrm{mM} \mathrm{MgCl}, 150 \mathrm{mM} \mathrm{NaCl}$, and $0.1 \mathrm{unit} / \mu \mathrm{L}$ of RNase $\mathrm{H}$ for the RNase $\mathrm{H}$ positive assays, at $37^{\circ} \mathrm{C}(50 \mathrm{mM}$ Tris- $\mathrm{HCl}, \mathrm{pH} 7.5)$. 
a

WT KRAS 5'- GAGCUGGUGGCGU-3'

G12V KRAS 5'-GGAGCU ' UUGGCG-3'

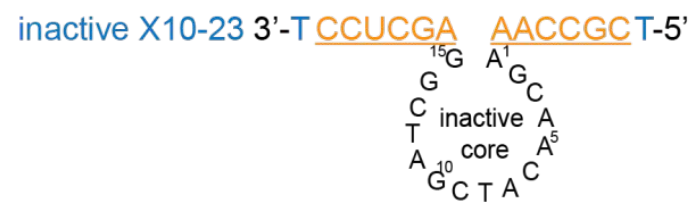

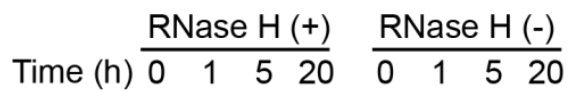

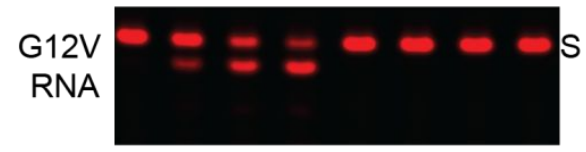

WT

RNA

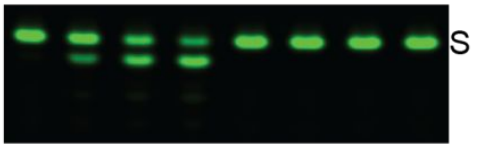

overlap

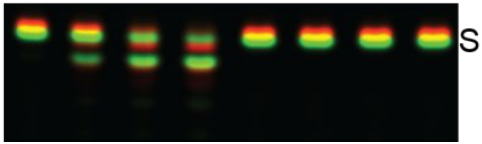

b

WT-KRAS 5'-GAGCUGGUGGCGU-3'

G12V-KRAS 5'-GGAGCUGUUGGCG-3'

Antisense 3'-TCCUCGACAACCGCT-5'

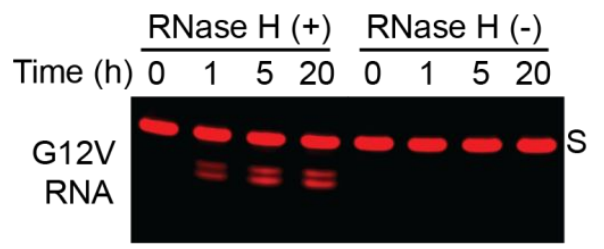

WT

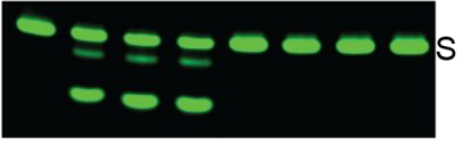

overlap 음통응

Figure S2. RNA cleavage activity in the presence of RNase $H$ with either X10-23 molecule consisting of an inactive core (a) or an ASO (b) targeting G12V substrate. Top panels: the base-pairing interactions of KRAS G12V cancer mutant or its corresponding wild-type (KRAS WT) mRNA with inactive G12V X10-23 and G12V antisense. Bottom panels: representative denaturing PAGE image showing the substrate degradation pattern in the presence or absence of RNase $\mathrm{H}$. mRNAs of KRAS G12V and KRAS WT are shown in red and green, respectively. All assays were performed in buffers containing equimolar $(1 \mu \mathrm{M})$ of total RNA substrate $(\mathrm{S})$ and inactive X10-23 molecule or ASO, $0.5 \mathrm{mM} \mathrm{MgCl}_{2}, 150 \mathrm{mM} \mathrm{NaCl}$, and 0.1 unit/ $\mu \mathrm{L}$ of RNase $\mathrm{H}$ for the RNase $\mathrm{H}$ positive assays, at $37^{\circ} \mathrm{C}(50 \mathrm{mM}$ Tris- $\mathrm{HCl}, \mathrm{pH} 7.5)$. 
a

$\mathrm{NCl}-\mathrm{H} 441+\mathrm{G} 12 \mathrm{~V}$ XNA-144h

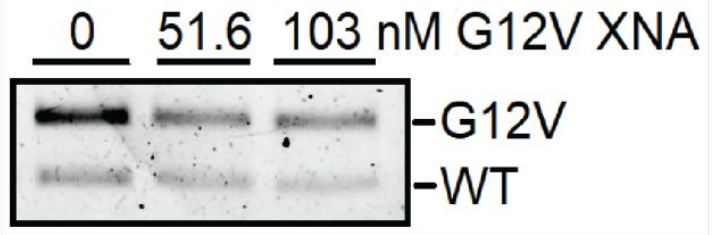

b

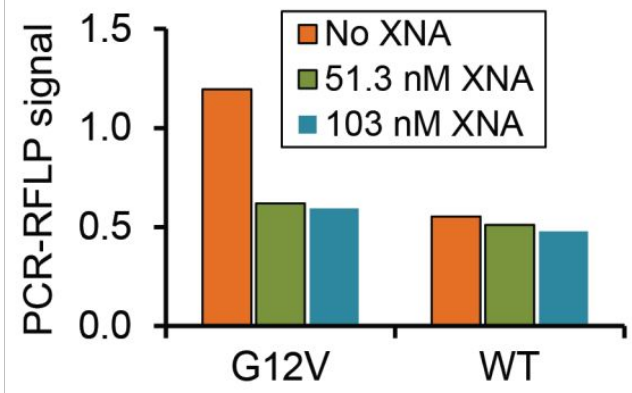

C

Plasm. control
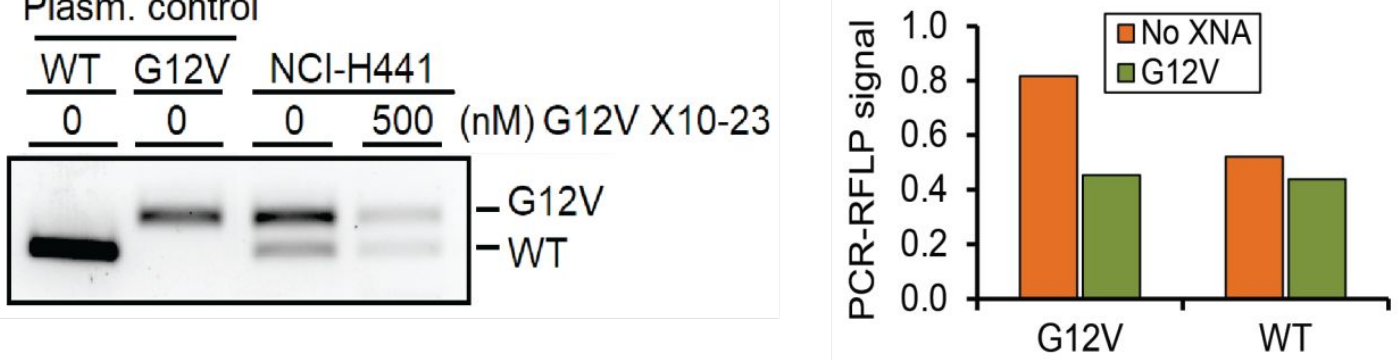

e

f

KRas plasmid

WT $\frac{\mathrm{G} 12 \mathrm{~V}}{\mathrm{NCl}-\mathrm{H} 441 \text { (KRas WT \& G12V) }}$
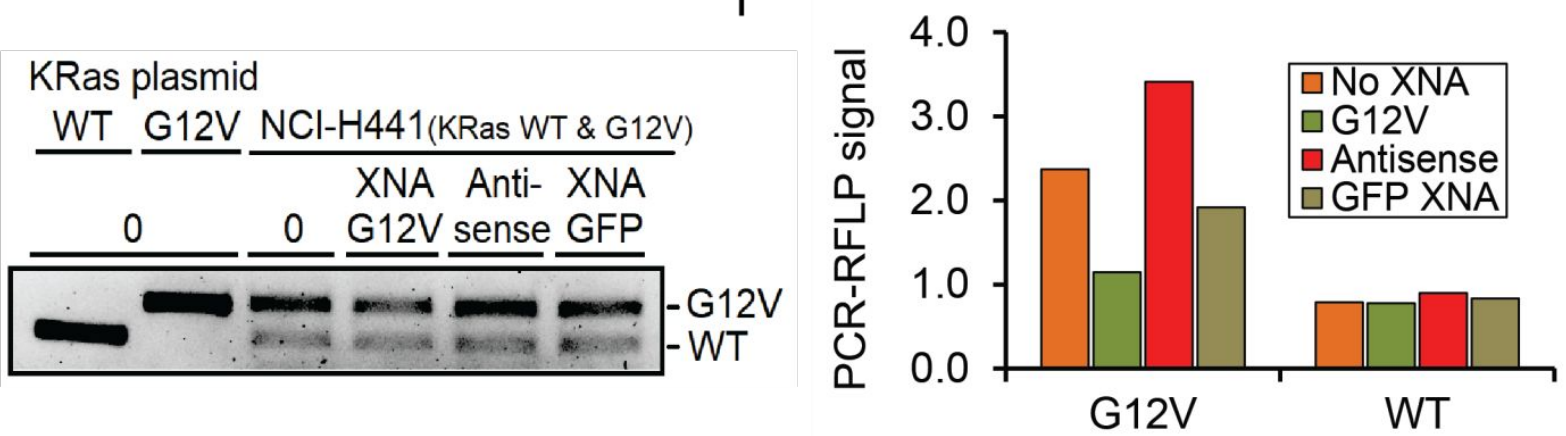

Figure S3. Assessment of G12V Kras X10-23 (XNA) targeting specificity by PCR-Restricted Fragment Length Polymorphism (PCR-RFLP) assay. Kras allele specific cleavage assay on cDNA from NCl-H441 cells containing both WT and G12V alleles. (a-d) NCl-H441 cells were treated with different concentration of G12V Kras X10-23 for $96 \mathrm{~h}$ or $144 \mathrm{~h}$ post transfection. DNAfree total RNA from untreated and treated were subjected to reverse transcription and PCR-RFLP assay. Two biological replicates $\mathrm{G} 12 \mathrm{~V}$ Kras $\mathrm{X} 10-23$ treated were performed with a biological representative shown. (e-f) KRAS allele-specific cleavage assay on cDNA from NCl-H441 cells treated with $16 \mu \mathrm{g}(306.8 \mathrm{nM}) \times 10-23$ targeting $\mathrm{G} 12 \mathrm{~V}, 16 \mu \mathrm{g}(604.3 \mathrm{nM})$ of the corresponding ASO, or $16 \mu \mathrm{g}(305.7 \mathrm{nM})$ of X10-23 targeting the GFP internal mRNA. Plasmids bearing WT or G12V KRAS sequence were used as controls. 


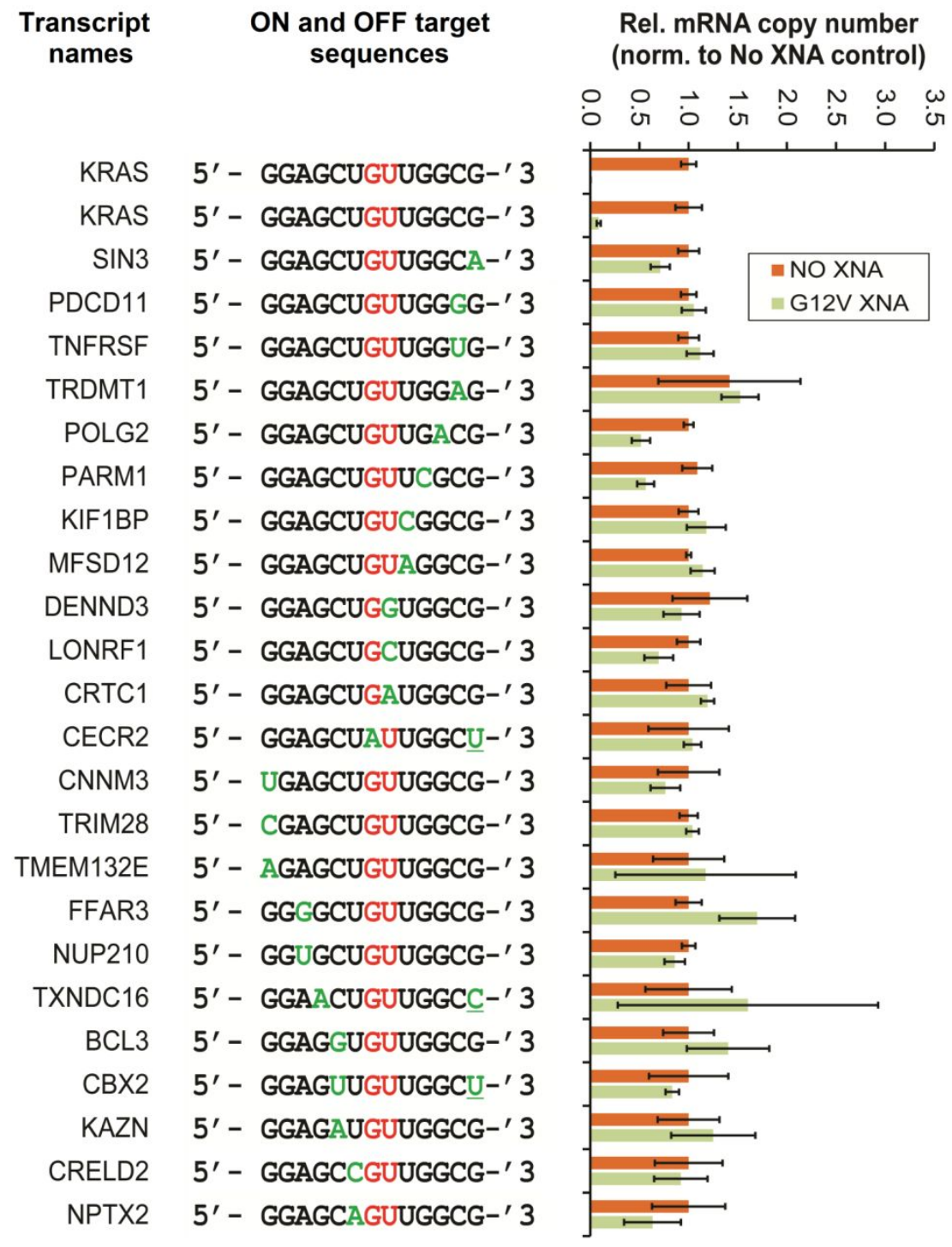

Figure S4. Survey off-targeting activity of G12V KRAS X10-23 (13nt FANA only binding arms). H441 cells were transfected with transfection reagent only (No X10-23 / 0) or $500 \mathrm{nM}$ G12V KRAS X10-23 for 96h. (Left), Transcript name; (Middle) the sequence of the intended (G12V KRAS target) and unintended targets (with various single mutation at the region downstream and upstream of GU cut site, green residues); (Right) RT-qPCR data shown on the bar graph represent relative mRNA copy number of both ON and OFF targets being assayed (horizontal axis). The values of each target on the horizontal axis were normalized to their corresponding negative control (untreated or No XNA). Error bars represent stardard deviation from the mean. The region harboring various mutations is where $\mathrm{X} 10-23$ binding arm in contact by complementary with potential targets (primer sequences listed on Table S1). DNA-free total RNA from cells (untreated and treated with G12V KRAS targeting X10-23) were subjected to cDNA synthesis and qPCR analysis using primers specific to twenty three (23) unintended targets which contain 1 or 2 mutations in the region flanking GU cut site of G12V X10-23. Gapdh which is served as a loading control. Representative of one biological and three technical replicates are shown except KRAS which has two biological replicates. 

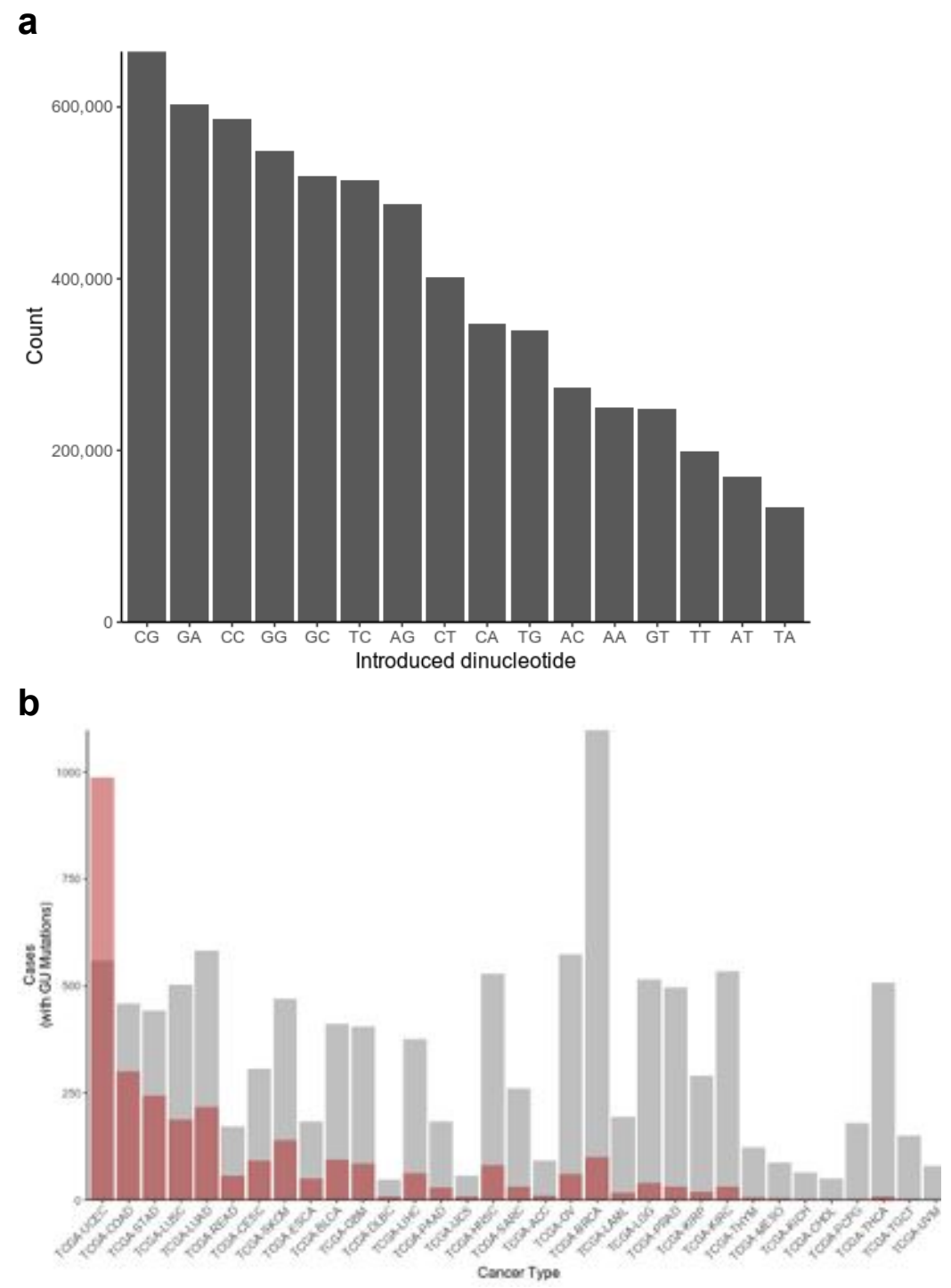

Figure S5. Analysis of acquired dinucleotide sequences from the Cancer Genome Atlas. (a) Count of dinucleotides introduced by single nucleotide polymorphisms in TCGA mutation data. For each gene impacted by a mutation, two introduced dinucleotides are counted: one consisting of the mutated position plus one base upstream, and one of the mutated positions plus one base downstream. (b) Prevalence of mutations inducing GU dinucleotides across cancer types. Gray bars show the total number of cancer cases in each TCGA project; red bars show the number of GU-inducing mutations present in those cases. GU mutation count can exceed case count if there are multiple GU mutations per case. 


\section{Supplementary tables}

Table S1: Primers used within the in vivo gene silencing mediated by $X 10-23$ including On/Off RNA targets)

\begin{tabular}{|c|c|c|}
\hline Primer ID & Sequence $\left(5 ’ \ldots-{ }^{\prime} 3\right)$ & Target \\
\hline oKN60F & ACC ATC TTC CAG GAG CGA GAT CCC TC & GAPDH \\
\hline oKN61R & TGC AGG AGG CAT TGC TGA TGA TCT TGA & GAPDH \\
\hline oKN111FC & ACCATGAGTG ATAACACTGC GGC & Standard \\
\hline oKN112RC & CGG TTC CCA ACG ATC AAG GC & Standard \\
\hline oKN424F & CAG CCA CAA CGT CTA TAT CAT GGC CG & EGFP \\
\hline oKN421R & GCT CAG GTA GTG GTT GTC GGG CAG & EGFP \\
\hline oKN458F & CGA GTT CGA GAT CGA GGG C & mCherry \\
\hline oKN459R & CAC CCT TGG TCA CCT TCA GC & mCherry \\
\hline oKN469F & GGC CTG CTG AAA ATG ACT GAA TAT AAA C & KRAS \\
\hline oKN470R & ACA AGA TTT ACC TCT ATT GTT GGA TCA TAT TCG & KRAS \\
\hline oKN505F & CCGGAC GCCACCTTCC & CNNM3 \\
\hline oKN506R & GGC GCA AGC GCA GCA G & CNNM3 \\
\hline oKN509F & AAAATCTACG TGATTGACGCCGAGAGG & DENND3 \\
\hline oKN510R & GCC TTT CCA AAT GGC GAC TTT CCC & DENND3 \\
\hline oKN517F & AGTGTGCTCT TTAACTTCGG AGAGAGATG & POLG2 \\
\hline oKN518R & CCT CGA GCT CCG CGT G & POLG2 \\
\hline oKNOT4F & CCAGGTTGCT CGTCTCTTTA AAAACCAG & SIN3 \\
\hline oKNOT4R & GGC CTC TGC GGC TTG TTG & SIN3 \\
\hline oKNOT5F & AGAAGGCAGA GAAGGAACTG TCCC & PDCD11 \\
\hline oKNOT5R & CAC GGC ACG GGC CTT C & PDCD11 \\
\hline oKNOT6F & GAGAGGCCA TAGCTGTCTG GC & TNFRSF \\
\hline oKNOT6R & GGT TCC TTT GTG GCA CTT GGT ACA G & TNFRSF \\
\hline oKNOT7F & GAACAGGATG GCAGGAGGGT TC & TRDMT1 \\
\hline oKNOT7R & TAA TTG CTA GGA TTA AAG GCT CCA AAA GCT AG & TRDMT1 \\
\hline oKNOT10F & AGAAGAGCGC GGAGCACC & PARM1 \\
\hline oKNOT10R & GGG CGG CAA GGA GTT TGC & PARM1 \\
\hline oKNOT14F & GCGGTGAGGC TGGCAG & KIF1BP \\
\hline oKNOT14R & AGA CCA CAA GAT ACC CAG GTT ATT CTG C & KIF1BP \\
\hline oKNOT15F & GGCGCGGC TGAGCTAC & MFSD12 \\
\hline oKNOT15R & TCG TAG CCC ACG AGC GG & MFSD12 \\
\hline oKNOT16F & CGGTTCTGG GAAGTGGGC & LONRF1 \\
\hline oKNOT16R & CAC TCG GGC CTG GCC & LONRF1 \\
\hline oKNOT17F & TGGGGACG CGTACTATGA GCAG & CRTC1 \\
\hline oKNOT17R & CAC TGT GGC TGG CGT ATC CC & CRTC1 \\
\hline oKNOT18F & GAGCGCCTG CGACCC & TRIM28 \\
\hline oKNOT18R & ATG TCT TTG GAG AAG CAC TGT TGC TTG & TRIM28 \\
\hline oKNOT19F & GCTGCCAGTC AGCTACCGC & TMEM132E \\
\hline oKNOT19R & ACC TGG CTG GTG CTG AAG G & TMEM132E \\
\hline oKNOT23F & GTGGTCCCGC TGATCATCAC C & FFAR3 \\
\hline oKNOT23R & CAC CGC AGA TAT AGC CCA CGA C & FFAR3 \\
\hline oKNOT25F & GCAGG GTCGCGGGAG & NUP210 \\
\hline oKNOT25R & CCA GCG TGA AGT TAA CGC GC & NUP210 \\
\hline oKNOT28F & TGGTTCCAGG CTACCCGG & TXNDC16 \\
\hline oKNOT28R & CTA CTG ATG ATC TAT GTT ATC TGC CCG GG & TXNDC16 \\
\hline
\end{tabular}




\begin{tabular}{|l|l|l|}
\hline oKNOT29F & GGGTCATCGA CATCCTGAGGG & BCL3 \\
\hline oKNOT29R & GGA GAC TGG GAG GGT GAG G & BCL3 \\
\hline oKNOT30F & TGTCCATGG GCCCGTCTG & CBX2 \\
\hline oKNOT30R & TGG AAA GGA GCC AAG CAG GG & CBX2 \\
\hline oKNOT31F & CTCCTGCGG GAAGAAGTGT CG & KAZN \\
\hline oKNOT31R & TCT TTG GCT CTG GCT AGC TCC TG & KAZN \\
\hline oKNOT33F & CGCCTGTGTG GATGTGGAC & CRELD2 \\
\hline oKNOT33R & TGC TCC CTC GCG TAG CC & CRELD2 \\
\hline oKNOT37F & AG TTTTTAATTC AGCTACTTTT TCCCCTCAGC & CECR2 \\
\hline OKNOT37R & GTG ATG AAG GAG AGG CGG AGA G & CECR2 \\
\hline $\begin{array}{l}\text { oKN484F } \\
\text { (PCR-RFLPA) }\end{array}$ & $\begin{array}{l}\text { ttattataaataatGACTGAATATAACTTGTGGTAGTTGGAcC } \\
\text { T }\end{array}$ & KRAS \\
\hline $\begin{array}{l}\text { oKN483R } \\
\text { (PCR-RFLPA) }\end{array}$ & $\begin{array}{l}\text { TCCTCTTGACCTGCTGTGTCG } \\
\text { [Sunaga et al. (2011) Mol Canc. Therapeutics] }\end{array}$ & KRAS \\
\hline
\end{tabular}

Table S2. Table of oligonucleotides

\begin{tabular}{|c|c|}
\hline Oligo Name & Sequence (5'-3') \\
\hline \multicolumn{2}{|c|}{ Oligonucleotides for KRAS inhibition } \\
\hline $\begin{array}{l}\text { X10-23 targeting } \\
\text { KRAS G12V }\end{array}$ & 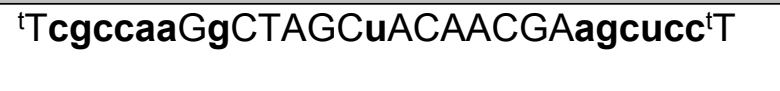 \\
\hline $\begin{array}{l}\text { X10-23 targeting } \\
\text { KRAS wild-type }\end{array}$ & ${ }^{\mathrm{t}}$ TacgccaGgCTAGCuACAACGAcagcuc'T \\
\hline $\begin{array}{l}\text { Inactive } \times 10-23 \\
\text { targeting KRAS } \\
\text { G12V }\end{array}$ & 'TcgccaagAGCAACATCGATCGGagcucc ${ }^{\mathrm{t} T}$ \\
\hline $\begin{array}{l}\text { Inactive X10-23 } \\
\text { targeting KRAS WT }\end{array}$ & 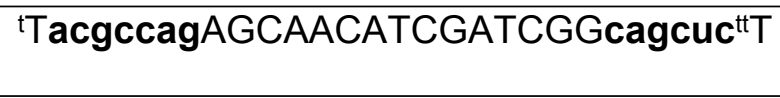 \\
\hline $\begin{array}{l}\text { X-antisense of } \\
\text { KRAS G12V }\end{array}$ & 'Tcgccaacagcucc $\mathrm{T}$ \\
\hline $\begin{array}{l}\text { X-antisense of } \\
\text { KRAS wild-type }\end{array}$ & 'Tacgccaccagcuc'T \\
\hline $\begin{array}{l}\text { KRAS G12V RNA } \\
\text { substrate }\end{array}$ & /5Cy5/rGrGrArGrCrUrGrUrUrGrGrCrG \\
\hline $\begin{array}{l}\text { KRAS wild-type } \\
\text { RNA }\end{array}$ & /5Cy5/rGrArGrCrUrGrGrUrGrGrCrGrU \\
\hline
\end{tabular}

Letters in bold lower cases denote FANA nucleotides; RNA residues are denoted by "r"; TNA residues are denoted by superscript " $\mathrm{t}$ ".

\section{References}

1. Noriaki Sunaga, David S. Shames, Luc Girard, et al., Mol. Canc. Therapeutics, 2011, 10, 336. 